\title{
Improvement of Optical Fiber Probing in Multiphase Systems, and the Possibility of Practical Application in Chemical Engineering Processes
}

\author{
Takayuki SaIto ${ }^{1}$, Katsuaki SATO ${ }^{2}$, Ayumi Nihei ${ }^{3}$ and \\ Hiroya Muramatsu ${ }^{2}$ \\ ${ }^{1}$ Research Institute of Green Science and Technology, Shizuoka University, 3-5-1 Johoku, Naka-ku, \\ Hamamatsu-shi, Shizuoka 432-8561, Japan \\ ${ }^{2}$ Graduate School of Science and Technology, Shizuoka University, 3-5-1 Johoku, Naka-ku, \\ Hamamatsu-shi, Shizuoka 432-8561, Japan \\ ${ }^{3}$ Graduate School of Engineering, Shizuoka University, 3-5-1 Johoku, Naka-ku, Hamamatsu-shi, \\ Shizuoka 432-8561, Japan
}

\begin{abstract}
Keywords: Optical Fiber Probing, Multiphase System, Bubble, Foam, Measurement Accuracy
Optical fiber probing (OFP) is a very useful and practical technique for investigating and monitoring multiphase systems, and it is particularly suitable for simultaneously measuring a bubble's/droplet's chord length, velocity and number density in an industrial-scale apparatus, as well as a laboratory-scale setup. Here, we outline the principles of OFP and propose several types of optical fiber probes that meet the requirements for particular purposes of the multiphase systems in chemical engineering processes. We describe measurement methods that use an optical fiber probe suitably tuned for liquid film and foam, as well as for bubble measurement. The basic measurement principle of OFP is very simple: the probe tip's detection of changes in the refraction indices from a gas phase to a liquid phase or vice versa. For example, to precisely measure a bubble's properties such as the chord length and velocity, it is necessary to determine the precise relationship between the process of the optical fiber probe's penetration into the bubble and the optical signal. Since the probing signal provides a variety of information due to the complicated interaction of the laser beams, optical fiber and gas-liquid interfaces, it is necessary to use both experimental and computational approaches in order to extract the physical meanings of the probe's signals. Using our own fully-3D ray-tracing simulator and well-arranged high-speed visualization setups, we discuss how to improve the measurement accuracy of OFP. We first computationally analyze the OFP signals under several penetration conditions, and we explain our recommendations regarding how to improve the accuracy of a single-tip optical fiber probe used for measurement in multiphase systems. On the basis of our present findings and the improvement in measurement accuracy, we then propose several applications of OFP to chemical engineering processes.
\end{abstract}

\section{Introduction}

Gas-liquid two-phase flows, one of the typical multiphase systems, are widely used in industrial processes such as bioreactors, chemical reactors and wastewater treatment plants. To carry out fundamental research into gas-liquid two-phase flows and for the development of the abovementioned plants, the dispersed phase of the flows must be measured, because the mechanisms of the flows are still unknown due to their nonlinear characteristics. In addition, to maintain the rated efficiency and high safety of the processes in such industrial-scale plants, careful monitoring of the gas-liquid two-phase flows is necessary.

Although electric conductivity probes have been studied, proposed and used frequently (e.g., Hibiki and Ishii, 2002),

Received on August 30, 2017; accepted on January 10, 2018 DOI: $10.1252 /$ jcej.17we283

Correspondence concerning this article should be addressed to T. Saito (E-mail address: saito.takayuki@shizuoka.ac.jp).

Presented at 3rd International Symposium on Multiscale Multiphase Process Engineering (MMPE2017), in Toyama, May 8-11, 2017 such probes present the problem of explosion protection for their application in some industrial plants. They also have no means of counteracting changes in the electric conductivity of the liquid phase. In contrast, optical fiber probing (OFP) (e.g., Abuaf et al., 1978; Cartellier, 1990, 1992; Cartellier and Achard, 1991; Cartellier and Barrau, 1998; Barrau et al., 1999; Mudde and Saito, 2001) is a promising, reliable and practical technique for measuring components of a dispersed phase flow (e.g., bubbles and droplets; OFP enables the simultaneous measurement of their chord length, velocity and number density for both fundamental and industrial purposes).

For the precise measurement of a bubble's/droplet's chord length (pierced chord length), velocity and number density (in other words, void fraction) by OFP, it is necessary to determine the exact relationship between the output optical signals from an optical fiber probe and the process of the probe's penetration into the measurement object. However, it is very difficult to determine this relationship through only experiments. Since the probing signal provides various types of information due to the complicated interaction of the laser beams (the light source for probing), the optical fiber and the gas-liquid interface, no previous research has 
succeeded in extracting the precise physical meanings of OFP signals.

An in-depth understanding of the relationship between the probing signal and the process of the probe's penetration into a measurement object is indispensable for improving the accuracy of OFP measurements. Ray-tracing computation is the most appropriate method for this purpose, and it is compatible with laser-based techniques in light of the directional sensitivity of laser beams. By using our own fully$3 \mathrm{D}$ ray-tracing simulator, we rendered complicated optical boundary conditions and trace ray-segment trajectories geometrically, and our findings revealed the physical meanings of the OFP signals.

On the basis of the computational and experimental results that we obtained using the computational simulator and a high-speed visualization setup, we have improved OFP measurement technology. In this paper, we summarize the OFP that we and our group developed, and we demonstrate the useful potential of this OFP in both fundamental and industrial applications for chemical engineering. These advances in OFP measurement technology will enable researchers and engineers studying multiphase flows to use OFP in further sophisticated ways.

\section{Principles of Optical Fiber Probing}

\subsection{OFP basics}

The basic measurement principle of optical fiber probing is very simple: the detection of changes in the refraction indices from a gas phase to a liquid phase (or vice versa) shown by the optical fiber probe's sensing tip. A plain optical fiber is composed of a cladding (an external layer for reflection) and a core (a guide for propagation). The refractive index of the cladding is slightly smaller than the core's, and some incident beams reach the sensing tip's surface after repeating the total reflection at the interface of the cladding and core, or transmission to the cladding, or emission to the outside of the optical fiber (as outlined in Figure 1).

When the sensing tip is positioned in a liquid phase (e.g., water), most of the beams reaching the sensing tip's surface are emitted in the liquid phase from the sensing tip's surface

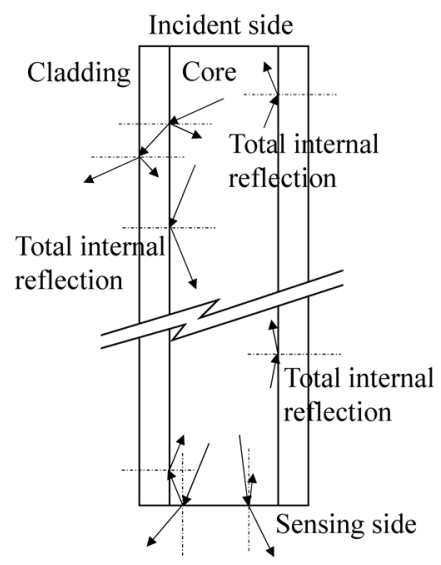

Fig. 1 Outline of the light propagation in an OFP setup (not to scale) due to the small difference between the refractive indices of the core and those of the liquid. Thus, few beams reflect at the sensing tip's surface and return to the incoming side end.

When the sensing tip is positioned in a gas phase (e.g., air), some of the beams reaching the sensing tip's surface are reflected at this surface due to the large difference between the refractive indices of the core and gas. Some of the beams reflecting at the sensing tip's surface thus return to the incoming side. The optical fiber probe's essential purpose is to detect changes in the refractive index around the sensing tip; in other words, OFP is based on detecting darkness or brightness (i.e., an on-off signal) at the incoming side. Based on this principle, most of the researchers in previous studies thought that at least a pair of optical fiber probes was necessary to simultaneously measure a dispersed phase's chord length and velocity.

\subsection{Principle of measurement through a single-tip optical fiber probe}

A few group have attempted the simultaneous measurement of velocity, chord length and number density of components of the dispersed phase such as bubbles and droplets by using a single-tip (mono-tip) optical fiber probe (e.g., Saito et al., 2009; Mizushima et al., 2013; Mizushima and Saito, 2015). The sensing tip of a single-tip optical fiber probe (S-TOP) developed by Saito et al. (2009) is ground into a wedge with an angle of $28-32^{\circ}$. The sensing tip is covered with a gas phase or liquid phase by degrees, and the returned light intensity thus changes by degrees (Figure 2).

For example in bubble measurement, when the summit of the wedge-shaped tip touches the frontal surface of the bubble, the intensity of the returned light increases, and as the tip penetrates the bubble's frontal surface more deeply, the increase in the area covered with the gas phase brings about an increase in the returned light intensity. The increase rate $\left(\mathrm{d} I_{\mathrm{R}} / \mathrm{d} t\right)$ of the returned light intensity $I_{\mathrm{R}}$ is proportional to the bubble velocity $U_{\mathrm{B}}$ (in a strict sense, the velocity of the bubble's frontal surface) (Mizushima and Saito, 2012),

$$
U_{\mathrm{B}}=\alpha \times \frac{\mathrm{d} I_{\mathrm{R}}}{\mathrm{d} t} \times \frac{1}{\left(I_{\mathrm{RGas}}-I_{\mathrm{RLiquid}}\right)}
$$

where $\alpha$ is a proportional coefficient determined by the surface tension and wettability (Mizushima and Saito, 2012). $\alpha$ is easily obtained by a preliminary experiment, in which

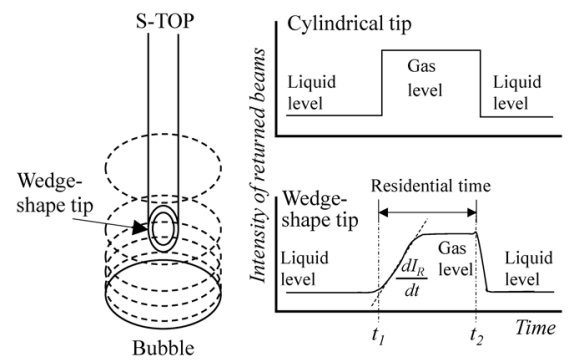

Fig. 2 Principle of S-TOP (single-tip optical fiber with a wedgeshaped tip) measurement. Relationship between the returned beams' intensity and the bubble and S-TOP 
S-TOP penetrates a gas-liquid interface from the liquidphase to gas-phase; in an air-water system at normal temperature, $\alpha$ is $0.0325 \mathrm{~mm}$. From this relationship, the bubble's velocity is calculated, and a pierced-chord length is calculated from this velocity and the residential time of the sensing tip in the bubble by the following equation:

$$
L_{\mathrm{D}}=U_{\mathrm{B}} \times\left(t_{2}-t_{1}\right)
$$

A local void fraction is obtained as the ratio of the sum of the residential time to the total measurement time (Mudde and Saito, 2001).

\subsection{Optical and data acquisition systems, and the configuration}

Figure 3 shows typical optical and data acquisition systems. The typical system consists of a laser diode (e.g., 54026-L, $635 \mathrm{~nm}$, Edmund Optics), an objective lens (e.g. OBL-40, NA=0.65, SIGMAKOKI Co., Ltd.), the optical fiber probe, a half mirror (e.g., CSMH-10-550, SIGMAKOKI Co., Ltd.), a polarization element (e.g., SPF-30C-32, linear polarization, Sigma Koki), and a photomultiplier (e.g., R928, Hamamatsu Photonics K.K.), a high-voltage supply (e.g., C9525, Hamamatsu Photonics K.K.), and an amplifier unit (e.g., C7319, Hamamatsu Photonics K.K.).

The photomultiplier is covered with an in-house-made electromagnetic shield. These optical elements are laid out on a rigid optical plate, and the optic axis is carefully adjusted in order to increase the signal-to-noise $(\mathrm{S} / \mathrm{N})$ ratio.

The data acquisition system consists of an A/D convertor and a personal computer (PC) equipped with executed data processing and data analysis software programs. The laser beams from the laser diode are focused on the incoming side of the optical fiber probe through the half-mirror and objective lens. The returned beams are detected by the photomultiplier through the half-mirror and a polarization element. The polarization element cuts the noise directly reaching the half-mirror by using the change of polarization planes occurring at the reflection at the half-mirror and other optical in-

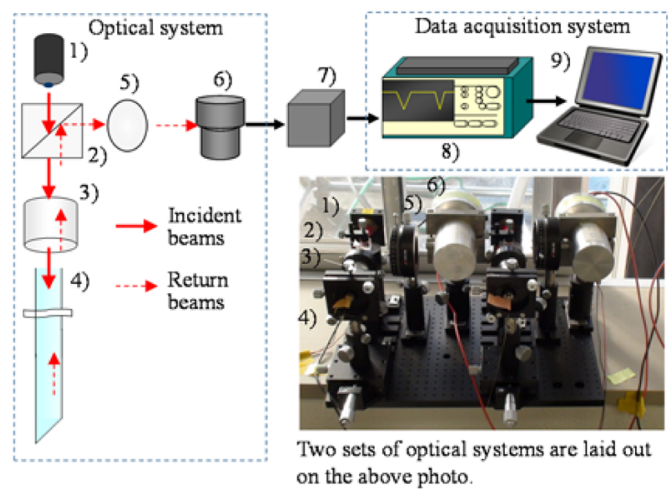

1) laser diode, 2) half mirror, 3) objective lens, 4) optical fiber probe, 5) polarization element, 6) electromagnetic shield (with a photomultiplier in this shield), 7) amplifier, 8) A/D converter, 9) personal computer.

Fig. 3 An example of optical and data acquisition systems, and their configuration terfaces. Thus, the beams returned from the sensing tip and the measurement object are selectively detected.

\section{Ray-Tracing Numerical Simulation}

\subsection{Outline of numerical simulation on the basis of a ray- tracing technique}

We have developed a full-3D ray-tracing numerical simulator that geometrically analyzes rays' paths and reveals the relationship between the optical signals of an S-TOP and the contact process of the S-TOP and an object. The rays can be translated into ray segments in the above-described optical system (Sakamoto and Saito, 2012a). Rendering complicated optical boundary conditions, the ray-segments trajectories are successfully traced geometrically with the use of our simulator; as a result, attribute types such as every ray's reflection point, every ray's energy at the reflection point, and every ray's polarization plane are accurately traced.

The rays' paths in the media and at the interfaces are calculated on the basis of Snell's law. The beams' energy and polarization are calculated on the basis of Fresnel's law. In addition, the optical boundary conditions are given not by polygons but rather by smooth functions; any unrealistic repetition of reflection (which occurs if the optical interfaces are rendered by polygons) is thus greatly suppressed. Using our simulator, we analyzed optical signals and observed promising phenomena hidden in the noise.

\subsection{Some numerical simulation results illustrating how to improve OFP accuracy}

2.2.1 Optical signals during an S-TOP's penetration process into a bubble The optical signals in bubble/droplet measurements obtained using an S-TOP usually look very noisy, due to the complex reflection and refraction at the deformable gas-liquid interface. However, noise-like peak signals include very useful phenomena for precise measurement by an S-TOP.

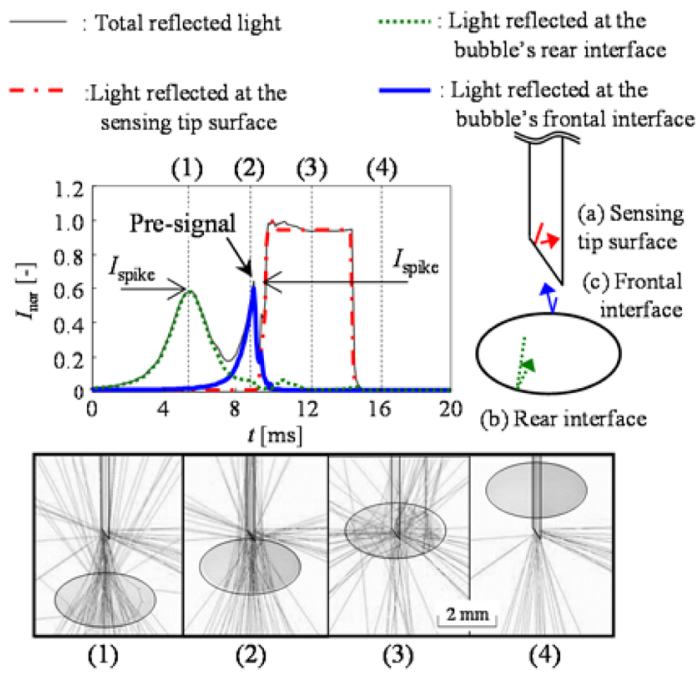

Fig. 4 A typical computational result of S-TOP signals in bubble measurement, and the corresponding rays' traces 
Figure 4 shows a typical computational result, i.e., the time evolution $t[\mathrm{~ms}]$ of optical signals $I_{\text {nor }}[-]$ (the intensity normalized by the maximum intensity) from an S-TOP (core dia. $150 \mu \mathrm{m}$; cladding thickness $25 \mu \mathrm{m}$; wedge angle $32^{\circ}$ ). Before the sensing tip of the S-TOP touches the frontal surface of an air bubble (major axis $3.4 \mathrm{~mm}$; minor axis $1.9 \mathrm{~mm}$ ) ascending in water, two noise-like peaks are observed (black line)

Two similar peaks often appear in real bubble measurement. The first peak (green line) arises from a phenomenon in which some of the beams reflecting at the bubble's rear surface re-enter the S-TOP. The second peak (blue line, named the 'pre-signal') arises from a phenomenon in which some of the beams reflecting at the bubble's frontal surface re-enter the S-TOP. The pre-signal clearly and strongly appears when the S-TOP touches a bubble at its center region in parallel with the bubble's minor axis (Mizushima et al., 2013).

Figures 5 and 6 illustrate the computational results regarding the influence of the S-TOP's touch position and the penetration angle (the angle between the S-TOP axis and the bubble's minor axis) on the normalized pre-signal intensity $I_{\text {spike }}[-]$, respectively.

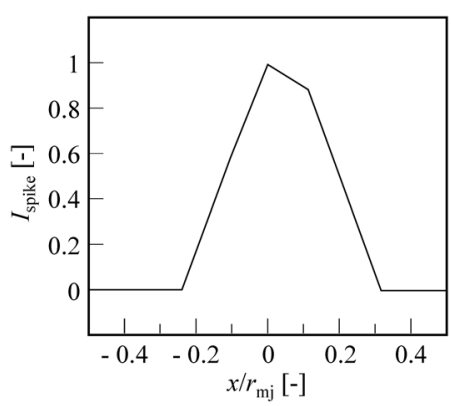

Fig. 5 Computational result of the relationship between normalized intensity of pre-signal $I_{\text {spike }}$ and normalized touch position $x / r_{\text {mj }}$

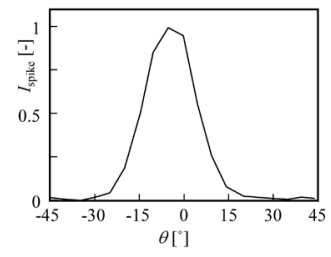

(a) $I_{\text {spike vs. } \theta}$

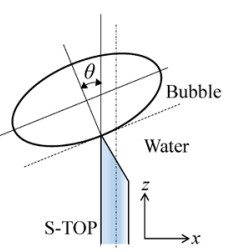

(c) Definition of the penetration angle $\theta$

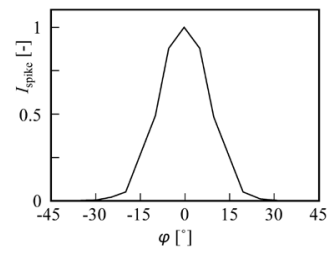

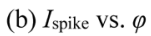

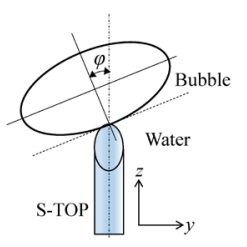

(d) Definition of the penetration angle $\varphi$
Fig. 6 Computational results of the relationship between the normalized intensity of the pre-signal $I_{\text {spike }}$ and the penetration angles $\theta$ and $\varphi$
Thus, the $I_{\text {spike }}$ is a function of the dimensionless touch position $x / r_{\mathrm{mj}}[-]$ and penetration angles $\theta\left[^{\circ}\right]$ and $\varphi\left[^{\circ}\right]$. The $I_{\text {spike }}$ takes the maximum at $x / r_{\text {mj }}=0$ (i.e., the center of the bubble), at $\theta=0$ (i.e., parallel with the bubble's minor axis) and at $\varphi=0$ (i.e., parallel with the bubble's minor axis). This characteristic of the pre-signal enables researchers to selectively and optionally extract signals at the center region of bubbles almost in parallel with the bubble's minor axis by the S-TOP ("Pre-signal threshold method," Mizushima et al., 2013). As a result, as demonstrated in the paper of Mizushima et al. (2013), by using this method in the data analysis, the random errors of bubble chord length and velocity due to an uncertain penetration of the S-TOP are markedly decreased.

2.2.2 Optical signals during an S-TOP's penetration into a thin liquid film Foam (i.e., foam cells) is one of the targets that is extremely difficult to be measured. Although a visualization method (Lu et al., 2013) and electric conductivity method (Xie et al., 2004) for foam measurement were proposed, reliable and accurate measurement techniques remain unavailable. The reason why measuring foam and foam cells is so difficult is that it requires the measurement of thin liquid films. A different measurement technique for thin liquid-film flow was proposed by Mitsuhashi and Saito (2010), the measurement technique, however, is not able to be applied to the foam measurement.

For the particular purpose of measuring foam and foam cells using an S-TOP, we computationally analyzed the optical signals when an S-TOP was penetrating a thin liquid film. The numerical results are plotted in Figure $7(\mathbf{a})$. As shown in the figure, the intensity of the total reflected light is the sum of mainly the light reflected at the sensing-tip surface, the light reflected at the liquid film's rear interface, and the light reflected at the liquid film's rear meniscus.

The intensity of the light reflected at the sensing tip surface takes the maximum when the sensing tip is exposed to a gas phase (e.g., air). When the sensing-tip peak touches the liquid film's frontal interface (Figure $7(\mathbf{b}-\mathbf{1})$ ), the intensity of the light reflected at the sensing tip surface decreases immediately; that is, the detection of this change provides the timing of when the sensing-tip peak touches the frontal interface of the liquid film. Similarly, when the sensing-tip peak touches the liquid film's rear interface (Figure 7(b-2)), the intensity of the light reflected at the liquid film's rear interface reaches its maximum; that is, the detection of this maximum indicates the time point at which the sensing-tip peak touches the rear interface of the liquid film. By applying the above knowledge, we are able to measure the thickness of the thin liquid film of a foam cell.

On the basis of other computational results, the wedgeshape's height $h$ must be smaller than the liquid film thickness in order to accurately measure the liquid film thickness with an S-TOP. In addition, to avoid bursting a thin liquid film, the S-TOP's surface must be super-hydrophilic for a water solution. 


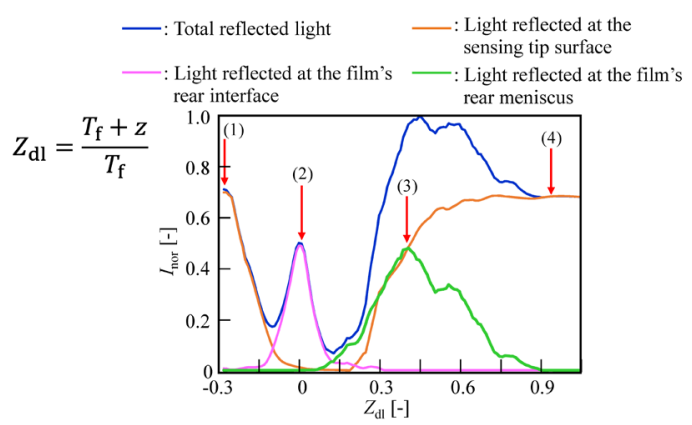

(a) Relationship between the normalized intensity $I_{\text {nor }}$ and the S-TOP's dimensionless distance $Z_{\mathrm{dl}}$

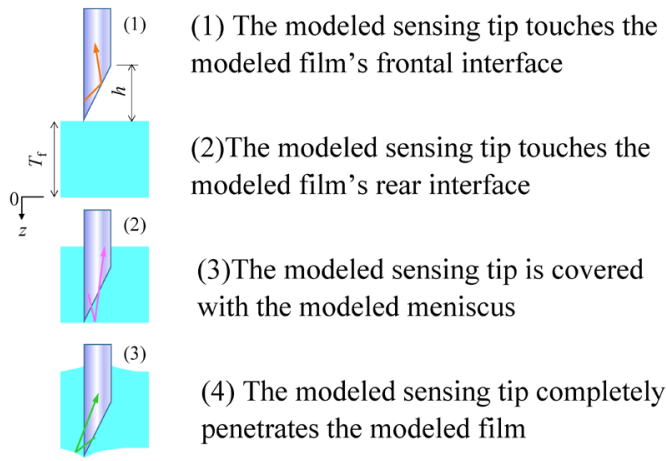

(b) An S-TOP's penetration process and the corresponding ray path

Fig. 7 Computational results of the normalized intensity of the S-TOP's optical signals during its penetration into a thin liquid film

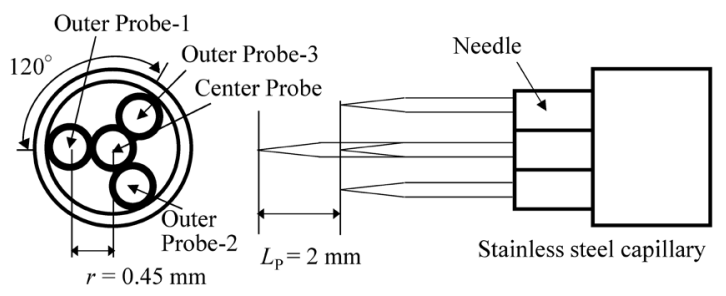

Fig. 8 Typical structure of a four-tip optical-fiber probe (F-TOP). The F-TOP is a bundle of four S-TOPs. The center probe is positioned at the center of the equilateral triangle composed by three outer probes. The radius of the equilateral triangle is decided in consideration of the presumed average diameter of the target bubbles. The gap $L_{\mathrm{p}}$ between the center probe apex and the outer probe apexes is decided in consideration of the presumed average diameter and orientation of the target bubbles

\section{Practical Use of the S-TOP and Other Types of Optical Fiber Probe}

\subsection{S-TOP measurement in a bubbly flow, and void fraction transportation}

The large-scale structure of a gas-liquid two-phase flow, which depends on the boundary condition, is essential to design a large-diameter bubble-column reactor. We considered a method to extract long-term fluctuations formed in a bubble column with an inner diameter of $380 \mathrm{~mm}$, on the basis of time-series point-wise void fractions. The spa-
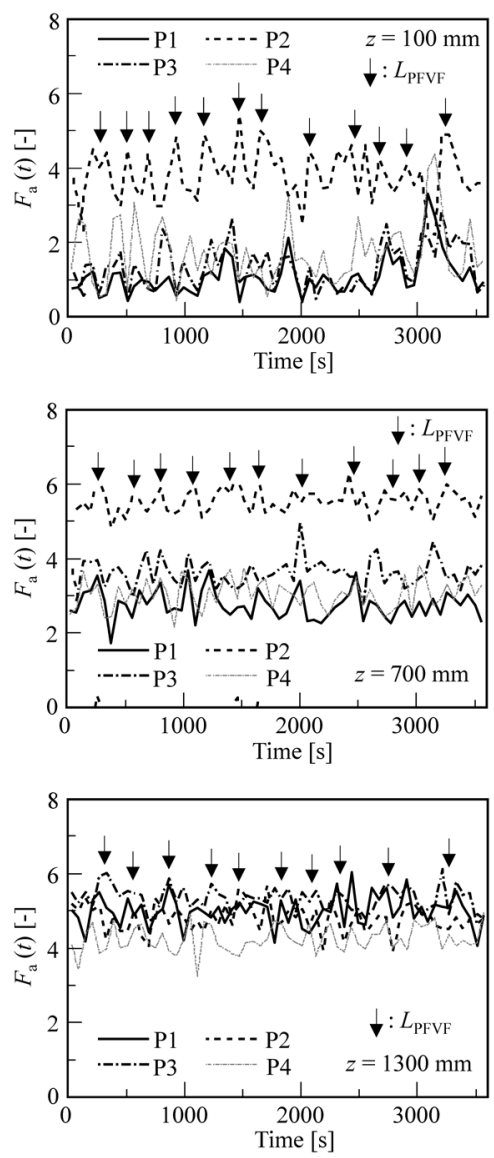

Fig. 9 Comparison of $F_{\mathrm{a}}(t)$ at four different positions.

P1: $(x, y)=(-95 \mathrm{~mm},-95 \mathrm{~mm}), \mathrm{P} 2:(x, y)=(-95 \mathrm{~mm}, 95 \mathrm{~mm})$, P3: $(x, y)=(95 \mathrm{~mm}, 95 \mathrm{~mm}), \mathrm{P} 4:(x, y)=(95 \mathrm{~mm},-95 \mathrm{~mm})$

tially averaged void fraction $F_{\mathrm{a}}(t)$ at an area $A_{\mathrm{a}}$ is defined as follows:

$$
F_{\mathrm{a}}(t)=\frac{\oint_{\mathrm{S}} f(x, y, t) \mathrm{d} S}{A_{\mathrm{a}}} \approx \frac{\int_{t_{1}}^{t_{2}} f_{\mathrm{a}}(x, y, t) \mathrm{d} t}{t_{2}-t_{1}}
$$

here, $f(x, y, t)$ represents the point-wise and spatial void fraction at a position $(x, y)$ and time $t ; A_{\mathrm{a}}$ the area which has characteristics similar to those of a void fraction; $f_{\mathrm{a}}$ the point-wise and temporal void fraction at a position $(x, y)$ and time $t ; t_{1}$ and $t_{2}$ the time interval which we are considering. On the basis of this relationship, we investigated longterm fluctuations (which reflect the large-scale structure of the bubbly flow) in the bubble column, using an S-TOP and a four-tip optical fiber probe (F-TOP; Figure 8) (Higuchi and Saito, 2010).

The row data obtained through the S-TOP and F-TOP were processed by our new algorithm (Sakamoto and Saito, 2012 b). Long-term fluctuations of the void fraction $\left(L_{\mathrm{PFVF}}\right)$ were successfully extracted through the above optical fiber probing. The $L_{\mathrm{PFVF}}$ faded away in accord with height of the flow (Higuchi and Saito, 2010) as shown in Figure 9; i.e. the large-scale structure of the bubbly flow in the vessel appeared to become weaker toward the vessel's upper surface. 


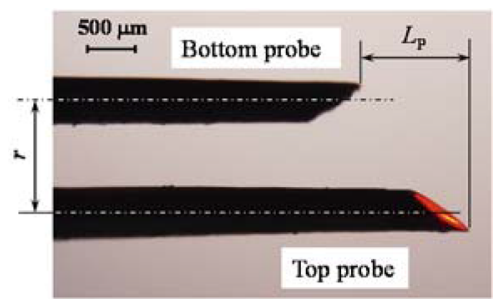

Fig. 10 A close-up photograph of the newly developed photoelectric optical fiber probe (POFP)

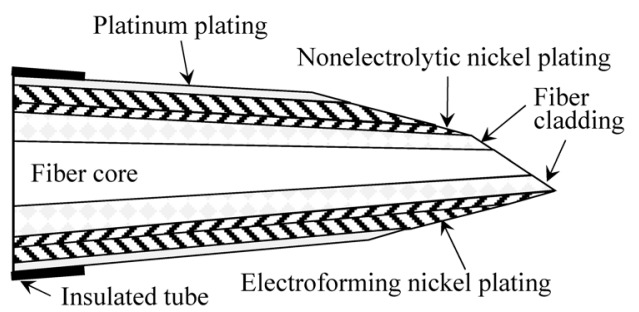

Fig. 11 Plated structure of the POFP

\subsection{Photoelectric optical fiber probe and concentration transportation}

To measure the point-wise $\mathrm{CO}_{2}$ concentration and its spatial and temporal concentration transportation as well as the bubble diameter, velocity and void fraction, we developed a photoelectric optical fiber probe (POFP): a pair of optical fiber probes plated with thin platinum and nickel layers (Figure 10) (Hanyu and Saito, 2010).

$L_{\mathrm{p}}$, the gap between the top probe and bottom probe, was decided in consideration of the bubble size and bubble orientation (Saito et al., 2010); $r$, the distance between the pair of probes, was decided in consideration of the bubble's wake structure and size (Saito and Toriu, 2015; Huang and Saito, 2015, 2017). The plated structure of the photoelectric optical fiber probe is shown in Figure 11.

The surface of the cladding of a plain optical fiber finedrawn with a $400-\mu \mathrm{m}$ tip diameter was non-electrolytically coated with a thin nickel layer (the first layer). The second layer of nickel was electrolytically plated on the first layer. The third layer of platinum was electrolytically plated on the second layer to improve the performance and to prevent oxidation of the electric sensor. Since the POFP measured the $\mathrm{CO}_{2}$ concentration from the electrical conductivity of the surrounding water dissolving $\mathrm{CO}_{2}$, the electric resistance from the sensor tip to a connection terminal had to be $<2 \Omega$.

To confirm the performance of this probe preliminarily, we measured the $\mathrm{pH}$ level in the wake of a single $\mathrm{CO}_{2}$ bubble by using the POFP and visualization based on the laser-induced fluorescence (LIF) of HPTS (a pH-sensitive and fluorescent substance: 8-hydroxypyrene-1,3,6-trisulfonic acid, trisodium salt; Coppeta and Rogers, 1998). In the above comparison, $\mathrm{pH}$ measurement by the POFP was rational, because HPTS is a pH-sensitive substance. Before the $\mathrm{pH}$ measurement, we obtained calibration curves between the electrical conductivity and $\mathrm{pH}$ level from 20 to $25^{\circ} \mathrm{C}$

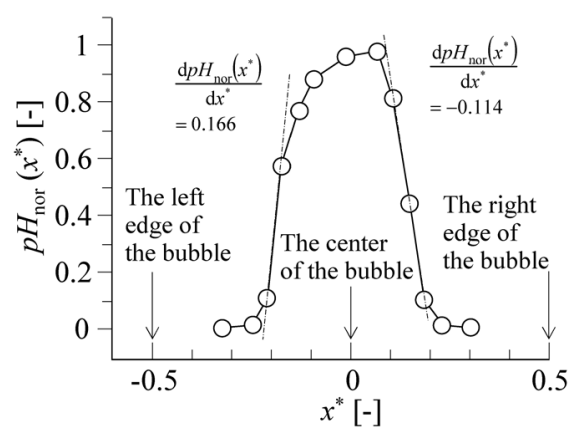

Fig. 12 A profile of the normalized $\mathrm{pH}$ measured by the POFP in the bubble wake

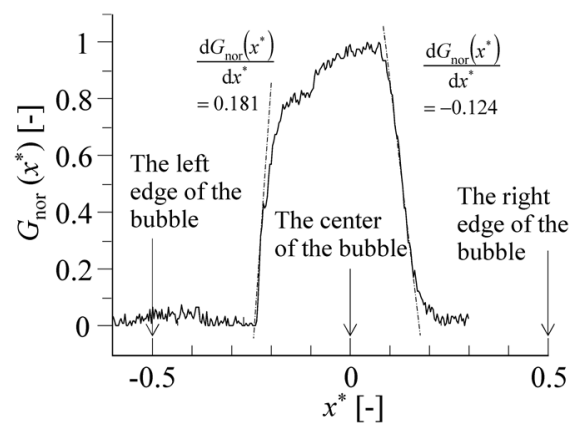

Fig. 13 A profile of the normalized gray scale by the visualization in the bubble wake

(Yamada and Saito, 2012).

Figures 12 and 13 illustrate our comparisons or the results obtained by both methods (Huang and Saito, 2017). Here, $\mathrm{pH}_{\text {nor }}\left(x^{*}\right)$ represents the normalized $\mathrm{pH}$ level in the wakes of a single $\mathrm{CO}_{2}$ bubble with 1.7-2.9-mm-dia. measured using the probe. $G_{\text {nor }}\left(x^{*}\right)$ represents the normalized gray scale in the bubble wake measured by the visualization, and $x^{*}$ represents the normalized position in the bubble's rear region.

The shapes of the profiles in Figures 12 and 13 are very similar; in addition, the gradients of the left-hand rising edge and the right-hand trailing edge in Figure 12 are very similar to those in Figure 13 (Huang and Saito, 2017). Since this POFP detected the $\mathrm{pH}$ level (i.e., the $\mathrm{CO}_{2}$ concentration) in areas that were much smaller than the bubbles in an intended bubble column (vessel dia. $380 \mathrm{~mm}$, vessel height: $1,500 \mathrm{~mm}$ ), we concluded that the POFP has enough spatial resolution to measure $\mathrm{CO}_{2}$ concentration in the bubble column.

We used two sets of POFPs to explore the relationship between the void fraction fluctuation of $\mathrm{CO}_{2}$ including bubbles and the $\mathrm{CO}_{2}$ concentration fluctuation of the surrounding liquid, as shown in Figure 14 (Yamada and Saito, 2012). The large-scale structure of a bubbly flow in a vessel is influenced by the boundary condition.

Higuchi and Saito (2010) discussed the long-term fluctuation of void fractions in a large-dia. bubble column $(380 \mathrm{~mm}$ inner diameter and $1,500 \mathrm{~mm}$ height). In consideration of the relationship between the temporal void-fraction fluc- 


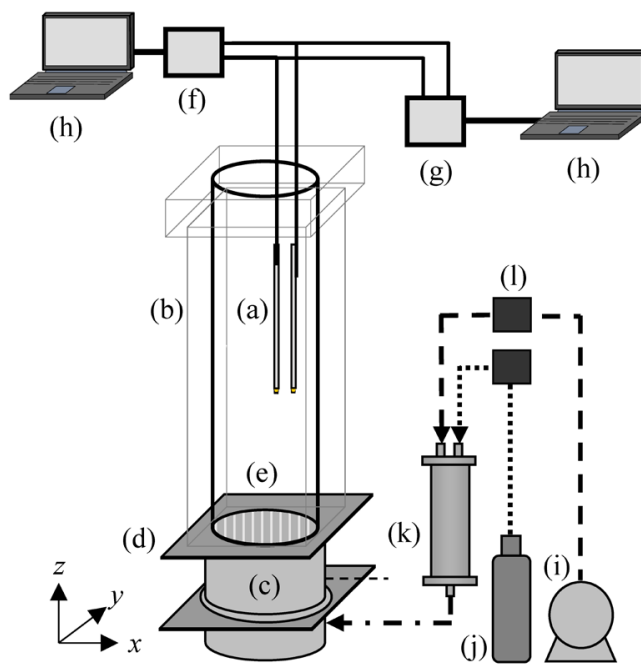

(a) POFP, (b) water jacket, (c) air chamber, (d) perforated plate, (e) acrylic bubble column, (f) differential amplifier circuit, (g) optical system, (h) PC, (i) air compressor, (j) $\mathrm{CO}_{2}$ gas cylinder, (k) gas mixer, (l) mass flow meter

Fig. 14 Experimental setup for a large-diameter bubble column experiment

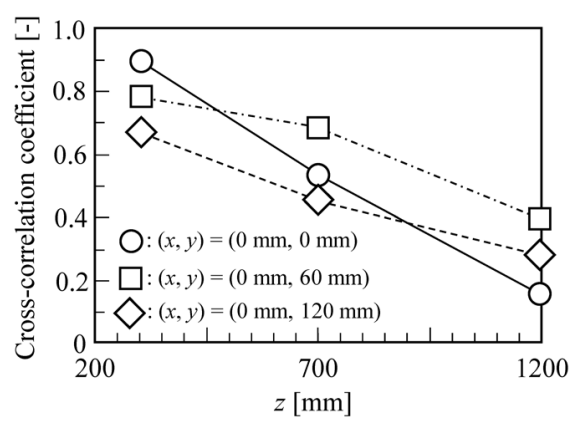

Fig. 15 Cross-correlation coefficients between the void fraction fluctuation and the concentration fluctuation

tuation and spatial void-fraction fluctuation, those authors showed the clear existence of a large-scale structure (i.e., a bubble swarm) in the lower part of the column, and its gradual disappearance toward the column surface. Yamada and Saito (2012), using the point-wise $\mathrm{CO}_{2}$ concentration measured by POFPs, obtained cross-correlation coefficients, and they considered the spatial scale of mass transportation (by a convective effect) in the bubble column. The cross-correlation coefficients in the bubble column (which were the same as Higuchi and Saito, 2010) are plotted in Figure 15.

The coefficient decreased toward the column surface. This tendency was very similar to the large-scale structure of the bubble swarms. Since the $\mathrm{CO}_{2}$ in the liquid phase was measured, this cross-correlation indirectly indicated the largescale structure of the liquid phase motion. Thus, the largescale structure of the liquid phase motion faded out toward the column surface. In light of this result, it appeared that the $\mathrm{CO}_{2}$ concentration in the liquid phase became spatially uniform toward the bubble column surface, in association with a spatially uniform dispersion of the bubbles. As de- scribed above, the POFP thus showed satisfactory performance and functions.

\subsection{Foam measurement using an S-TOP}

We developed an S-TOP and signal processing for foam measurement on the basis of the results described above in Section 2.2.2. We carried out two simple experiments. The experiment shown in Figure $\mathbf{1 6}$ was performed to measure thin liquid films, and the other experiment, in which the liquid film stand was replaced with a foam vessel (Figure 17), was performed to measure foam cells.

The liquid examined in both experiments was a solution composed of water, polyvinyl alcohol and a surfactant, and its refractive index was 1.333 . The tip diameter of the S-TOP was $10 \mu \mathrm{m}$, and the tip was ground into an angle of $32^{\circ}$. The S-TOP surface was chemically coated with a super-hydrophilic material (silane coupling agent) to avoid bursting the thin liquid films. As shown in Figure 18, two target thin liquid films were set up in parallel and kept tight at a $10-\mathrm{mm}$ distance. Each target thin liquid film was held by eight thin liquid films, as shown in Figue 19; the eight thin liquid films were held by four fine fishing lines (outer diameter: $0.090 \mathrm{~mm}$ ) strained on four stainless rods.

Using the above-described technique, we visualized the penetration process of the S-TOP clearly without disturbance of the fishing lines. The S-TOP mounted on a six-axis precision auto-drive micro-stage was moved downward at a constant and arbitrary rate, and the S-TOP then penetrated the target thin liquid films perpendicularly. The penetration process was filmed by a high-speed video camera synchronized with the micro-stage, and the optical signal was converted into electric signal through the photomultiplier and stored in the digital oscilloscope.

Figure 20 is a typical snapshot of the penetration process of the S-TOP. The target thin liquid films did not burst and the penetration process was clearly visualized.

From this result, an S-TOP that is appropriately surfaceengineered can avoid bursting the thin liquid films of foam cells. The S-TOP's signal corresponding to the penetration process in Figure 20 is plotted in Figure 21.

Two signals of A and B detecting the thin liquid films are clearly observed; on the basis of the explanation above in Section 3.2.2, by analyzing the raw data between $A_{1}$ and $A_{2}$ and that between $B_{1}$ and $B_{2}$, the thickness of the liquid films are calculated. The average film thickness measured by the S-TOP was $26.5 \mu \mathrm{m}$; the average thickness calculated from the liquid film's mass and surface area was $25.6 \mu \mathrm{m}$. The difference between them was $3.4 \%$ of the thickness. The interval of the two thin liquid films was calculated from the time lag $\Delta t$ and the descending speed of the S-TOP; the average film interval was $10.0 \mathrm{~mm}$ measured by the S-TOP, and $10.1 \mathrm{~mm}$ measured by the visualization.

The above S-TOP measurement was found to be satisfactory when used in a field test of foam measurement in a new wastewater treatment system, as described below. 


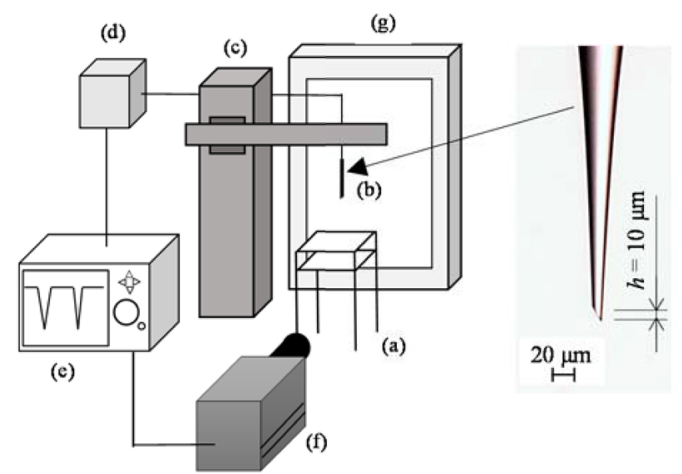

(a) film stand, (b) S-TOP, (c) six-axis precision auto-drive microstage, (d) optical system, (e) digital oscilloscope (A/D convertor), (f) high-speed video camera, (g) light source

Fig. 16 Experimental setup used in the thin liquid film measurement

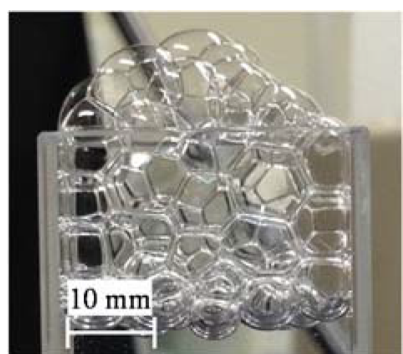

Fig. 17 A foam vessel and foam used for foam cell measurement

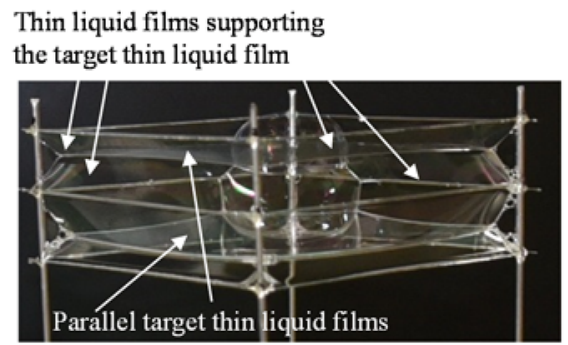

Fig. 18 Two target thin liquid films held on the film stand

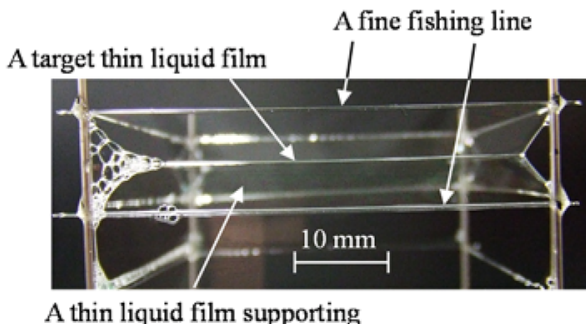

A thin liquid film supporting the target thin liquid film

Fig. 19 Details of the upper target thin liquid film

\subsection{R\&D of a new wastewater treatment system using the solidification reaction of milk fats and proteins through ozonation}

We have been researching and developing a new wastewater treatment system using the ozonation of milk fats and proteins (Sato and Saito, 2013) for the specific purpose of dealing with new and tightened regulations in Japan regard-

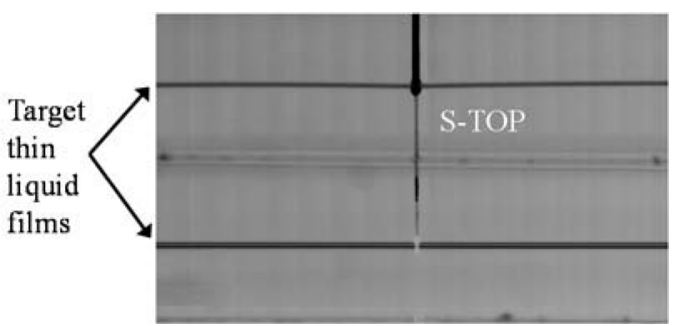

Fig. 20 A typical close-up snapshot of an S-TOP's penetration process of a target thin liquid film

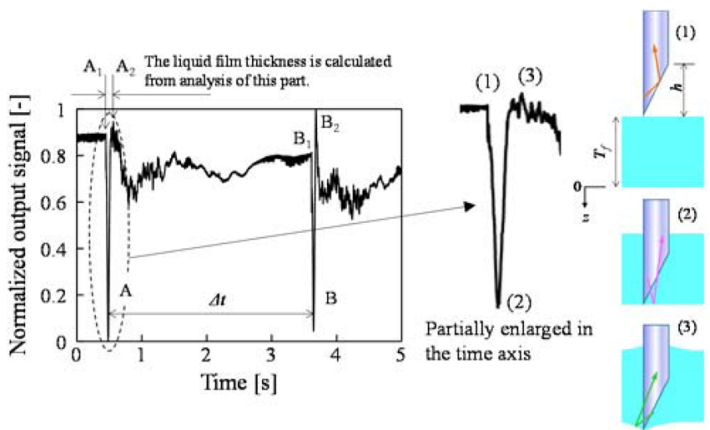

Fig. 21 S-TOP's signal corresponding to the penetration process shown in Figure 20

ing wastewater discharged from small-to-medium-size dairy farms. It is too difficult for the farm managers to economically treat the farms' wastewater including milk fats and proteins. Some treatment systems using ozonation have been proposed, but they were not suited for use in dairy farms due to high operating costs, low user-friendliness and high maintenance costs.

We proposed a new ozonation process enforcing the partial polymerization of unsaturated fatty acids as well as the simple decomposition of the acids into small molecules. Whitish solid-like products formed from the polymerized fatty acids (i.e., ozonide) and the insoluble proteins were adsorbed to the ozone-bubble surfaces (i.e., flotation). Thus, the chemical reaction between the ozone and the milk fats and proteins and the physical reaction between these products and bubble surfaces must be revealed. The efficiency of these processes depends strongly on the bubbly flows in the new system.

We accumulated data (3D profiles of the bubble number density, bubble properties and liquid-phase velocity, etc.) regarding the bubbly flows by using a laboratory experimental setup (Figure 22).

A photograph of a typical bubbly flow setup in the new wastewater treatment system and a photo of the thick and dense ozonide foam formed at the bubble column surface are shown in Figure 23. The ozonide foam was measured with an S-TOP as described above in Section 3.3.

Integrating the results of the laboratory experiments and our knowledge and know-how regarding bubbly flows, and taking into account the properties of wastewater discharged from dairy farms, we designed a wastewater-treatment system that has been used in field tests at a dairy farm in Japan 

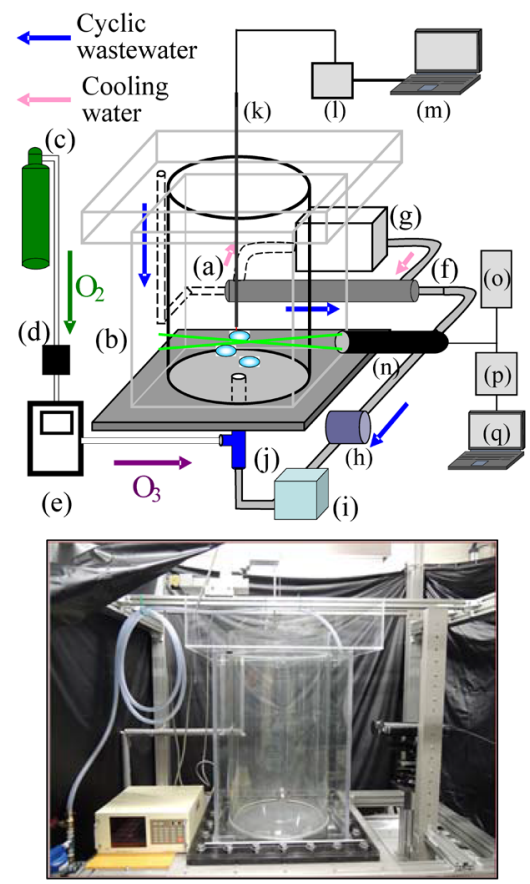

(a) acrylic bubble column $(520 \mathrm{~mm}$ in inner diameter, $1040 \mathrm{~mm}$ in height), (b) acrylic water jacket, (c) $\mathrm{O}_{2}$ cylinder, (d) gas flow meter and controller, (e) ozone generator, (f) heat exchanger, (g) chiller, (h) circulation pump, (i) electromagnetic flow meter, (j) ejector, (k) S-TOP, (l) pptical system and A/D convertor, (m) PC, (n) LDV (Laser Doppler Velocimeter) probe, (o) Ar+ laser system, (p) LDV system, (q) PC

Fig. 22 Experimental setup used in the laboratory experiments

at about $1,000 \mathrm{~m}$ elevation. The new system (Figure 24) is very simple and user-friendly.

The LDV was too fragile to use in the field tests, and we therefore used an S-TOP to measure bubbles in the bubble column and the ozonide foam (Nihei and Saito, 2015). On the basis of the data accumulated in our laboratory experiments, we found that the bubble and foam measurements were satisfactory and useful for discussing the performance of the field-test system.

The optical system and data acquisition system are shown in Figure 25. The S-TOP we used to measure the ozonide foam was mounted on a triaxial automatic stage and moved downward at an arbitrary speed and position.

Typical ozonide foam that formed at the bubble column surface is shown in Figure 26, and the S-TOP measuring the foam is shown in Figure 27. The large-scale and smallscale structure of the bubble swarms and the properties of the bubbles in the bubble column were very similar to those observed in the laboratory experiments.

The foam-cell's chord length in the vertical direction (i.e., the depth from the foam surface) is plotted in Figure 28. The foam-cell's chord length tended to decrease toward the foam bottom. This tendency was the same as that observed in our laboratory experiments, but the average foam-cell's chord length was larger than that seen in the laboratory experiments by approx. $10 \%$.

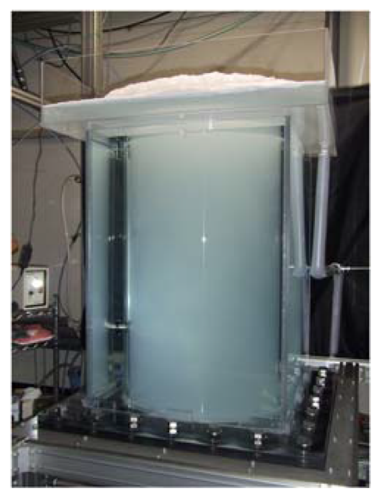

(a) Overall picture of a bubbly flow during wastewater treatment

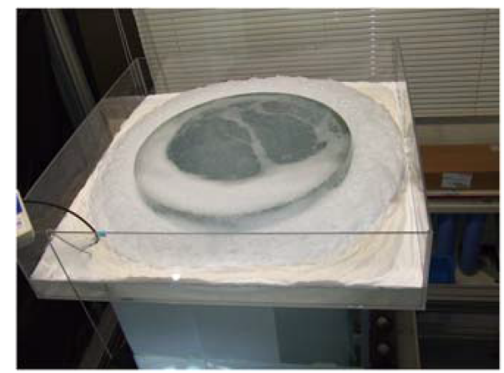

(b) Thick and dense ozonide foam formed at the bubble column surface

Fig. 23 Treatment of model wastewater that includes milk in the laboratory experiments

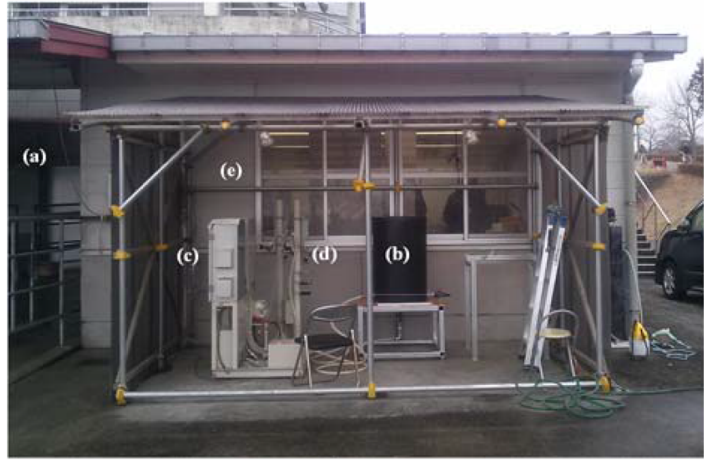

(a) milking room, (b) bubble column (550 $\mathrm{mm}$ in inner diameter, and $860 \mathrm{~mm}$ in height) for wastewater treatment, (c) controlling board, (d) gas flow meter, (e) measuring room

Fig. 24 Outline of the field test at a dairy farm

We speculate that this difference in foam-cell's chord length was due to the lower atmospheric pressure at the test site. Thus, we successfully accumulated data needed for the design of a commercial system and for the estimation of maintenance costs.

\section{Conclusions}

We described the principle of OFP measurement technology and how to computationally analyze the optical signals of the OFP system. The principle and the hardware are very 


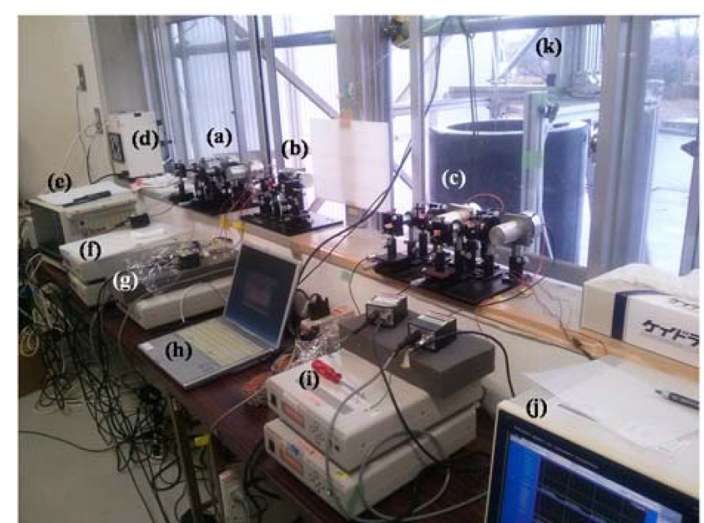

(a) S-TOP optics for ozonide foam measurement, (b) spare optics, (c) S-TOP optics for bubble measurement in the bubble column, (d) ozone generator, (e) high-speed A/D convertor, (f) controllers for automatic stages, (g) high-voltage supply for the S-TOP for the foam, (h) PC, (i) high-voltage supply for the STOP for the bubbles in the bubble column, (j) PC, (k) triaxial automatic stage for the S-TOP for foam measurement.

Fig. 25 Measurement system for the S-TOPs in the field test

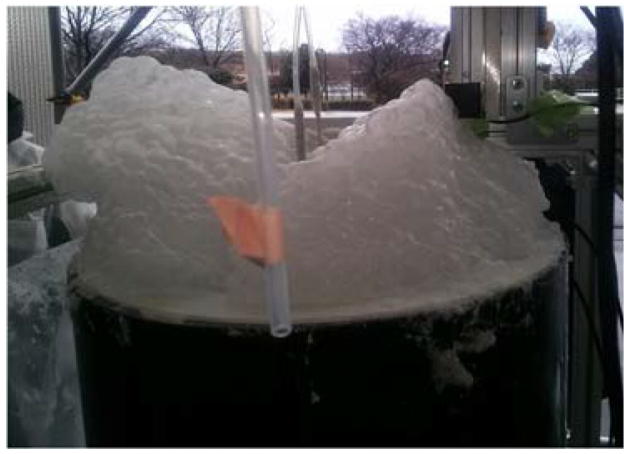

Fig. 26 Typical ozonide foam in the field test

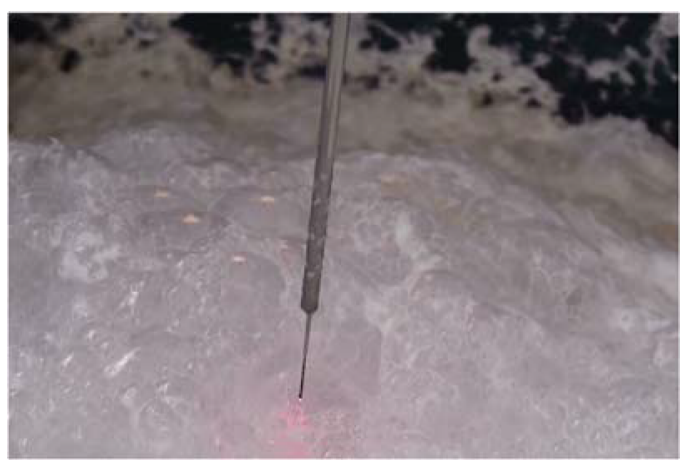

Fig. 27 S-TOP measuring the ozonide foam in the field test

simple, and the OFP measurement technology is promising in for multiphase flow research; however, the optical signals and its physical meanings are very difficult to understand. Carrying out computational and experimental activities, we revealed a small part of the hidden potential of the OFP measurement technology. On the basis of the results described herein, we proposed some ideas for using OFP more

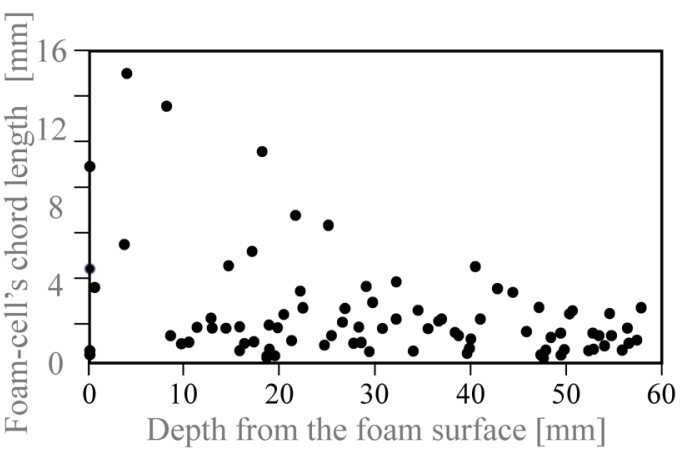

Fig. 28 Relationship between the foam-cell's chord length and its vertical position from the foam surface, measured through the $\mathrm{S}$-TOP in the field test

reliably and for more sophisticated purposes: the pre-signal threshold method showing how to detect the piercing position of the S-TOP; the F-TOP method explaining how to extract the large-scale structure of a bubbly flow; the POFP method for measuring the $\mathrm{CO}_{2}$ concentration; and the use of an S-TOP for measuring foam cells and their film thickness.

We successfully obtained data that can be used for the design of a commercial system by using an S-TOP developed for foam measurement and the results of our laboratory experiments and knowledge about bubbly flows as well as OFP measurement. We also designed a new wastewatertreatment system for field tests at a dairy farm. In effect, our findings demonstrate that optical fiber probing is useful, reliable and applicable to both laboratory experiments and field tests. We believe that optical fiber probing has great potential for many types of measurement.

\section{Nomenclature}

$A_{\mathrm{a}} \quad=$ area of spatially averaged void fraction

$\left[\mathrm{mm}^{2}\right]$

$f(x, y, t)=$ point-wise and spatial void fraction at a position $(x, y)$ and time $t$

$f_{\mathrm{a}}(x, y, t)=$ point-wise and temporal void fraction at a position

$(x, y)$ and time $t$

$F_{\mathrm{a}} \quad=$ spatially averaged void fraction at $A_{\mathrm{a}}$

$G_{\text {nor }}=$ normalized gray scale in the wakes of a single $\mathrm{CO}_{2}$ bubble

$[-]$

$[-]$

$h \quad=$ height of wedge-shape tip of the S-TOP $\quad[\mathrm{mm}]$ or $[\mu \mathrm{m}]$

$I_{\text {nor }} \quad=$ returned light intensity normalized by the maximum intensity

$I_{\mathrm{R}} \quad=$ returned light intensity

$[\mathrm{V}]$ or $[-$

$I_{\mathrm{RGas}}=$ returned light intensity when the OFP sensing tip positioned in a gas phase

$I_{\text {RLiquid }}=$ returned light intensity when the OFP sensing tip positioned in a liquid phase

$[\mathrm{V}]$ or $[-]$

$I_{\text {spike }} \quad=$ normalized intensity of pre-signal

$L_{\mathrm{D}} \quad=$ bubble chord length pierced by the OFP

$[-]$

$L_{\mathrm{D}} \quad=$ gap between OFPs

$[\mathrm{mm}]$

$\mathrm{pH}_{\text {nor }}=$ normalized $\mathrm{pH}$ level in the wakes of a single $\mathrm{CO}_{2}$ bubble $[-]$

$r \quad=$ distance between OFPs $\quad[\mathrm{mm}]$ or $[\mu \mathrm{m}]$

$r_{\mathrm{mj}}=$ bubble radius $\quad[\mathrm{mm}]$

$t=$ time [s] or [ms]

$t_{1} \quad=$ start time of touching the bubble's surface by the 
OFP's sensing tip

$t_{2}=$ end time of touching the bubble's surface by the OFP's sensing tip

$$
\text { OFP's sensing tip }
$$

$t_{\mathrm{A}}=$ start time of the time interval considered in integration of temporal void fraction

$\begin{aligned} t_{\mathrm{B}}= & \text { end time of the time interval considered in } \\ & \text { integration of temporal void fraction }\end{aligned}$

$\begin{aligned} t_{\mathrm{B}}= & \text { end time of the time interval consider } \\ & \text { integration of temporal void fraction }\end{aligned}$

$T_{\mathrm{f}} \quad=$ liquid film thickness

$[\mathrm{s}]$ or $[\mathrm{ms}$

$U_{\mathrm{B}} \quad=$ bubble velocity or velocity of the bubble's frontal surface

$[\mathrm{m} / \mathrm{s}]$ or $[\mathrm{mm} / \mathrm{s}]$

$x=$ the horizontal position

$[\mathrm{mm}]$

$x^{*}=$ normalized position of the POFP in the bubble rear region

$x / r_{\mathrm{mj}}=$ dimensionless touch position

$z \quad=$ vertical position

$Z_{\mathrm{dl}} \quad=$ dimensionless vertical position

$[\mathrm{mm}]$ or $[\mu \mathrm{m}]$

$=$ proportional coefficient

$=$ penetration angle

$=$ penetration angle

\section{Literature Cited}

Abuaf, N., O. C. Jones Jr. and A. Zimmer; "Optical Probe for Local Void Fraction and Interface Velocity Measurements," Rev. Sci. Instrum., 49, 1090-1094 (1978)

Cartellier, A.; "Optical Probes for Local Void Fraction Measurements: Characterization of Performance," Rev. Sci. Instrum., 61, 874-886 (1990)

Cartellier, A.; "Simultaneous Void Fraction Measurement, Bubble Velocity, and Size Estimate Using a Single Optical Probe in Gas-Liquid Two-Phase Flows," Rev. Sci. Instrum., 63, 5442-5452 (1992)

Cartellier, A. and J. L. Achard; "Local Phase Detection Probes in Fluid/ Fluid Two-Phase Flows," Rev. Sci. Instrum., 62, 279-303 (1991)

Cartellier, A. and E. Barrau; "Monofibre Optical Probes for Gas Detection and Gas Velocity Measurements: Conical Probes," Int. J. Multiph. Flow, 24, 1265-1294 (1998)

Coppeta, J. and C. Rogers; "Dual Emission Laser Induced Fluorescence for Direct Planar Scalar Behavior Measurements," Exp. Fluids, 25, $1-15$ (1998)

Barrau, E., N. Riviere, C. Poupot and A. Cartellier; "Single and Double Optical Probes in Air-Water Two-Phase Flows: Real Time Signal Processing and Sensor Performance," Int. J. Multiph. Flow, 25, 229-256 (1999)

Hanyu, K. and T. Saito; "Dynamical Mass Transfer Process of a $\mathrm{CO}_{2}$ Bubble Measured by Using LIF/HPTS Visualization and Photoelectric Probing," Can. J. Chem. Eng., 88, 551-560 (2010)

Hibiki, T. and M. Ishii; "Interfacial Area Concentration of Bubbly Flow Systems," Chem. Eng. Sci., 57, 3967-3977 (2002)

Higuchi, M. and T. Saito; "Quantitative Characterizations of LongPeriod fluctuations in a Large-Diameter Bubble Column Based on Point-Wise Void Fraction Measurements," Chem. Eng. J., 160, 284-292 (2010)

Huang, J. and T. Saito; "Influence of Bubble-Surface Contamination on Instantaneous Mass Transfer," Chem. Eng. Technol., 38, 1947-1954 (2015)

Huang, J. and T. Saito; "Influences of Gas-Liquid Interface Contamination on Bubble Motions, Bubble Wakes, and Instantaneous Mass Transfer," Chem. Eng. Sci., 157, 182-199 (2017)

Lu, K., R. Li, Z. Wua, K. Hou, X. Du and Y. Zhao; "Wall Effect on Rising Foam Drainage and Its Application to Foam Separation," Sep. Purif. Technol., 118, 710-715 (2013)

Mitsuhashi, Y. and T. Saito; "Measurement of a Liquid Film Inside the Steam-Water Two-Fluid Nozzle Using An Optical Fiber Probe (in Japanese)," Trans. JSRAE, 27, 149-160 (2010)

Mizushima, Y. and T. Saito; "Detection Method of a Position Pierced by a Single-Tip Optical Fibre Probe in Bubble Measurement," Meas. Sci. Technol., 23, 085308 (2012)

Mizushima, Y., A. Sakamoto and T. Saito; "Measurement Technique of Bubble Velocity and Diameter in a Bubble Column via Single-Tip Optical-Fiber Probing with Judgment of the Pierced Position and Angle," Chem. Eng. Sci., 100, 98-104 (2013)

Mizushima, Y. and T. Saito; "Nonlinear Bubble Nucleation and Growth Following Filament and White-Light Continuum Generation Induced by a Single-Shot Femtosecond Laser Pulse into Dielectrics Based on Consideration of the Time Scale," Appl. Phys. Lett., 107, 114102 (2015)

Mudde, R. F. and T. Saito; "Hydrodynamical Similarities between Bubble Column and Bubbly Pipe Flow," J. Fluid Mech., 437, 203-228 (2001)

Nihei, A. and T. Saito; "A Novel Measurement Technique Based on a Single-Tip Optical Fiber Probe for Dense Foam," Proceedings of 12th Gas-Liquid-Solid Reactor Engineering (2015)

Saito, T., K. Matsuda, Y. Ozawa, S. Oishi and S. Aoshima; "Measurement of Tiny Droplets Using a Newly Developed Optical Fibre Probe Micro-Fabricated by a Femtosecond Pulse Laser," Meas. Sci. Technol., 20, 114002 (2009)

Saito, T., K. Sakakibara, Y. Miyamoto and M. Yamada; "A Study of Surfactant Effects on the Liquid-Phase Motion around a ZigzaggingAscent Bubble Using a Recursive Cross Correlation PIV," Chem. Eng. J., 158, 39-50 (2010)

Saito, T. and M. Toriu; "Effects of a Bubble and the Surrounding Liquid Motions on the Instantaneous Mass Transfer across the Gas-Liquid Interface," Chem. Eng. J., 265, 164-175 (2015)

Sakamoto, A. and T. Saito; "Computational Analysis of Responses of a Wedge-Shaped Optical Fiber Probe in Bubble Measurement," Rev. Sci. Instrum., 83, 075107 (2012a)

Sakamoto, A. and T. Saito; "Robust Algorithms for Quantifying Noisy Signals of Optical Fiber Probes Employed in Industrial-Scale Practical Bubbly Flows," Int. J. Multiph. Flow, 41, 77-90 (2012b)

Sato, K. and T. Saito; "A Newly Developed Wastewater Treatment by Using Solidification Reaction of Milk Fats and Proteins through Ozonation," Chemical Engineering Transactions, The Italian Association of Chemical Engineering, 32, 1-6 (2013)

Xie, W., S. J. Neethling and J. J. Cilliers; "A Novel Approach for Estimating the Average Bubble Size for Foams Flowing in Vertical Columns," Chem. Eng. Sci., 59, 81-86 (2004)

Yamada, M. and T. Saito; "A Newly Developed Photoelectric Optical Fiber Probe for Simultaneous Measurements of a $\mathrm{CO}_{2}$ Bubble Chord Length, Velocity, and Void Fraction and the Local $\mathrm{CO}_{2}$ Concentration in the Surrounding Liquid," Flow Meas. Instrum., 27, 8-19 (2012) 